\title{
Development of High Efficient and Low Toxic Oil Spill Dispersants Based on Sorbitol Derivants Nonionic Surfactants and Glycolipid Biosurfactants
}

\author{
Dandan Song ${ }^{1,2}$, Shengkang Liang ${ }^{1,2 *}$, Qianqian Zhang ${ }^{1,2}$, Jiangtao Wang ${ }^{1,2}$, Limei Yan ${ }^{1,2}$ \\ ${ }^{1}$ Key Laboratory of Marine Chemistry Theory and Technology, Ministry of Education, Qingdao, China; ${ }^{2}$ College of Chemistry and \\ Chemical Engineering, Ocean University of China, Qingdao, China. \\ Email: *liangsk@ouc.edu.cn
}

Received 2013

\begin{abstract}
Dispersants, usually blending with several surfactants and a solvent, are used to enhance oil spill dispersion as small droplets in water column. Although there is growing acceptance of dispersants as a counter measure to marine oil spills around the world, the two major issues with the dispersants are their toxicity to marine life and dispersion effectiveness (DE) for crude-oil, especially for heavy oil. To develop more efficient and less toxic dispersants, two kinds of sorbitol derivant nonionic surfactant (polysorbate 85 and sorbeth-40 tetraoleate), two kinds of glycolipid biosurfactants (rhamnolipid and sophorolipid) and less toxic solvent ethylene glycol butyl ether were chosen in this study, and two dispersant formulations were optimized by uniform design methods. Effects of dispersant-to-oil ratio, temperature, salinity and $\mathrm{pH}$ on the performance of the two optimized dispersants were investigated. The two dispersants had high dispersion effectiveness (DE) for heavy crude oil, while both dispersants keep high DE at the dispersant-to-oil ratio below 1:25 and the temperature above $5^{\circ} \mathrm{C}$. In addition, the two dispersants also performed well in a wide range of salinity and $\mathrm{pH}$ values. Finally, toxicity tests revealed that the two dispersants showed low toxicity to two kinds of fish (Danio rerio and Microgobius gulosus).
\end{abstract}

Keywords: Oil Spill Dispersant; Formula Uniform Design; Baffled Flask Test; Effectiveness; Toxicity

\section{Introduction}

Increasing exploitation, production, transportation, and storage of oil around the world have caused more oil spills in the oceans [1-3]. When an oil spill occurs, the spills will most likely spread over a large area under gravitational, surface tension, and viscous forces if the quick response is not initiated and the environmental and economic effects can be devastating, such as sensitive shorelines, local wildlife and plant life [4]. So, careful spill response technologies are needed to minimize the damage of oil spills at sea. Except for mechanical response and in situ burning, the application of chemical dispersants is an efficient mean of reducing the environmental and economic impact of spilled oil [5].

Dispersants are usually sprayed onto oil slicks to accelerate the dispersion of oil from the sea surface into the water column. A typical dispersant may consist of three or more surfactants and solvent. Surfactants usually have oil-soluble hydrocarbon chains and water-soluble groups, are partially soluble in both oil and water, and the free

${ }^{*}$ Corresponding author. energy of the system is minimized when surfactants are present at the oil-water interface [6]. Surfactant blends show high dispersant effectiveness when compared with individuals, means synergistic agonistic interactions between surfactants $[7,8]$ Nevertheless, not all surfactant compositions are suitable for consisting dispersants to disperse spilled oil effectively, and many of the effective ones have the drawbacks of being toxic and/or non-biodegradable [9]. During the 1970s and 80s, many countries resisted the use of dispersants. This was mainly because of high toxicity of dispersants, which contains some aromatics compounds in the solvents. For example, the Torrey Canyon spill, in which the use of toxic dispersant led to subsequent widespread environmental damage and an impression that dispersant use only adds to the problem. [10]. During the course of years, the chemical compositions of dispersants have been changing from especially toxic ones to not so toxic products. Many of modern dispersants are very low in toxicity - an order of magnitude lower than many common household products, which could be related use of nonionic surfactant and solvents such as the glycol ethers and water in- 
stead of aromatic compounds [11,12]. In recently, lowertoxicity and more effective dispersants have led to broadly acceptance of dispersant use. Oil Spill Intelligence once reported that 36 out of 149 countries rely on dispersant use as their primary response option and another 62 consider it a secondary option [13].

Many dispersants are effective in dispersing easily dispersed oils. However, the effectiveness can become highly disparate when testing more viscous products of heavy oils or weathered crudes, especially in cold water, and higher energy is required for breaking up the treated higher viscosity slick into small droplets $[14,15]$.

Mixture design and response surface methods have been used to optimize oil spill dispersants [7]. In this study, uniform design method was used to optimize dispersants formulations. Dispersant effectiveness was conducted according to an improved dispersant testing protocol, named the Baffled Flask Test (BFT) [16]. Low toxic and biodegradable sorbitol derivants nonionic surfactants and glycolipid biosurfactants were selected as variables to compose oil spill dispersants formulations. The aims of present work are to achieve optimized formulations of dispersant by determining the effectiveness in dispersing QHD32-6 crude oil and to investigate the effects of some important factors, such as dispersant-tooil ratio (DOR), temperature, salinity and $\mathrm{pH}$, on dispersion effectiveness.

\section{Materials and Methods}

\subsection{Materials}

\subsubsection{Crude oil and Seawater}

The tested heavy crude oil was submitted by China National Offshore Oil Corp. Its general physicochemical properties are listed in Table 1. The seawater used was conducted from East China Sea and its salinity, $\mathrm{pH}$, and temperature are 32 per thousand, 8.02 , and $15^{\circ} \mathrm{C}$, respectively.

\subsubsection{Surfactants and Solvent}

The sorbitol derivants nonionic surfactants were polysorbate 85 (Tween 85) and sorbeth-40 tetraoleate (GO440) purchased from Nikko Chemicals Co., Ltd. (Shanghai,

Table 1. Physicochemical properties of QHD32-6 crude oil used.

\begin{tabular}{ll}
\hline Properties & Value \\
\hline API gravity at $15^{\circ} \mathrm{C}$ & $15.7 \sim 16.5$ \\
Viscosity at $50^{\circ} \mathrm{C}(\mathrm{mPa} \cdot \mathrm{s}$, ) & $408 \sim 634$ \\
Asphaltenes content (wt. \%) & 3.7 \\
Paraffin content (wt. \%) & $2.26 \sim 3.28$ \\
\hline
\end{tabular}

China), respectively. And the biosurfactants were sophorolipids (SLs) and rhamnolipids (RLs), produced by Candida bombicola ATCC22214 grown on glucose/ rapeseed oil culture medium and Pseudomonas aeruginosa O-2-2 grown on rapeseed oil culture medium in the laboratory, respectively. The culture conditions, purification, and characterization of the two glycolipid biosurfactants have been reported in our early research $[17,18]$. The properties of four surfactants are shown in Table 2 . The solvent of disperstant was ethylene glycol butyl ether (2-butoxyethanol) and purchased from GuangCheng Chemical Co., Ltd. (Tianjin, China)

\subsection{Methods}

\subsubsection{Uniform Design Method}

A uniform design (UD) seeks design points that are uniformly scattered on the domain, was proposed by Fang [19] based on quasi-Monte Carlo method or numbertheoretic method. It has been popular since 1980. In UD results, the levels of all variables or factors included in chemical experiments are changed continuously, allows the largest possible amount of levels for each factor, that is, the number of levels could be equal to the number of experiment runs. In this study, dispersant formulation contains 50 percent of surfactants, and the other half was 2-butoxyethanol as solvent. Four surfactants used as four factors in UD, different compositions influenced the dispersion effectiveness. In order to get more information from experiment, each factor took 24 levels, $\mathrm{U}_{24}\left(24^{4}\right)$ was chosen for experimental design that was listed in Table 3.

\subsubsection{Dispersion Experiments}

Baffled Flask Test (BFT) method was performed in determining dispersant effectiveness $[16,20]$. A stock solution of dispersant-oil mixture in dichloromethane (DCM) was prepared. Specific volume of stock standard solution was added to $30 \mathrm{~mL}$ seawater in a $125 \mathrm{~mL}$ separatory funnel and extracted with DCM. The final extract was adjusted to $25 \mathrm{~mL}$ with DCM. For QHD32-6 crude oil,

Table 2. Properties of four surfactants used.

\begin{tabular}{cccc}
\hline Surfactants $^{\mathrm{a}}$ & HLB $^{\mathrm{b}}$ & $\begin{array}{c}\text { Minimal surface tension } \\
(\mathrm{mN} / \mathrm{m})\end{array}$ & $\begin{array}{c}\mathrm{CMC}^{\mathrm{c}} \\
(\mathrm{mg} / \mathrm{L})\end{array}$ \\
\hline SLs & $12-13$ & 30 & 30 \\
RLa & $22-24$ & 29 & 40 \\
Tween 85 & 11.0 & 43 & 0.5 \\
GO440 & 12.5 & unknown & unknown \\
\hline
\end{tabular}

a. The abbreviations of SLs, RLa, Tween 85, and GO440 represent sophorolipids, rhamnolipids, polysorbate 85 , and sorbeth-40 tetraoleate surfactants, respectively; b and c correspond to hydrophilic-liphophilic-balance and Critical Micelle Concentrations, respectively. 
Table 3. Factors and levels of surfactants in the oil dispersants (Abbreviations as in Table 2, the solvent is 2-butoxyethanol).

\begin{tabular}{|c|c|c|c|c|c|c|}
\hline \multirow{2}{*}{ No. } & \multicolumn{5}{|c|}{ Dispersant formulation composition } & \multirow{2}{*}{$\begin{array}{l}\text { HLB value of } \\
\text { dispersant }\end{array}$} \\
\hline & SLs (\%) & RLs (\%) & $\begin{array}{c}\text { Tween } 85 \\
\text { (\%) }\end{array}$ & $\begin{array}{l}\text { GO440 } \\
(\%)\end{array}$ & $\begin{array}{l}\text { Solvent } \\
\text { (\%) }\end{array}$ & \\
\hline 1 & 36.25 & 2.88 & 7.15 & 3.71 & 50.00 & 12.47 \\
\hline 2 & 30.15 & 12.30 & 6.10 & 1.43 & 50.00 & 14.35 \\
\hline 3 & 26.45 & 6.30 & 3.44 & 13.80 & 50.00 & 13.33 \\
\hline 4 & 23.70 & 20.05 & 0.28 & 5.95 & 50.00 & 16.06 \\
\hline 5 & 21.40 & 2.33 & 16.25 & 10.05 & 50.00 & 12.25 \\
\hline 6 & 19.40 & 13.05 & 10.35 & 7.15 & 50.00 & 14.46 \\
\hline 7 & 17.65 & 1.68 & 5.55 & 25.15 & 50.00 & 12.48 \\
\hline 8 & 16.05 & 17.10 & 1.08 & 15.75 & 50.00 & 15.55 \\
\hline 9 & 14.60 & 13.25 & 21.85 & 0.28 & 50.00 & 14.21 \\
\hline 10 & 13.30 & 9.15 & 13.60 & 13.95 & 50.00 & 13.70 \\
\hline 11 & 12.05 & 26.05 & 7.45 & 4.45 & 50.00 & 17.11 \\
\hline 12 & 10.85 & 10.05 & 2.10 & 27.00 & 50.00 & 14.24 \\
\hline 13 & 9.75 & 9.45 & 27.25 & 3.515 & 50.00 & 13.37 \\
\hline 14 & 8.75 & 3.58 & 16.70 & 21.00 & 50.00 & 12.60 \\
\hline 15 & 7.75 & 19.85 & 9.40 & 13.00 & 50.00 & 15.91 \\
\hline 16 & 6.80 & 0.83 & 3.28 & 39.10 & 50.00 & 12.49 \\
\hline 17 & 5.85 & 5.30 & 33.10 & 5.75 & 50.00 & 12.46 \\
\hline 18 & 5.00 & 23.60 & 19.85 & 1.57 & 50.00 & 16.34 \\
\hline 19 & 4.16 & 12.20 & 11.45 & 22.20 & 50.00 & 14.43 \\
\hline 20 & 3.35 & 34.15 & 4.60 & 7.90 & 50.00 & 18.82 \\
\hline 21 & 2.56 & 1.57 & 40.60 & 5.30 & 50.00 & 11.56 \\
\hline 22 & 1.80 & 17.25 & 23.10 & 7.85 & 50.00 & 15.07 \\
\hline 23 & 1.07 & 3.68 & 13.60 & 31.65 & 50.00 & 12.78 \\
\hline 24 & 0.35 & 24.50 & 6.05 & 19.05 & 50.00 & 16.96 \\
\hline
\end{tabular}

the six calibration concentrations obtained were 0.0926 , $0.2036,0.3980,0.7034,0.9256$ and $1.2958 \mathrm{~g} / \mathrm{L}$. Then the absorbance of oil standard solutions relative to a DCM blank was measured from 340 to $400 \mathrm{~nm}$ using a UV-VIS Spectrophotometer (UV Prove 2.0, Shimadzu). The area under the absorbance vs. wavelength curve was automatically integrated between 340 and $400 \mathrm{~nm}$. For dispersant effectiveness analysis, first, $100 \mathrm{~mL}$ seawater, equilibrated at the desired temperature, was added to the baffled flask, and then $90 \mathrm{mg}$ oil was dispensed directly onto the surface of seawater. Finally, the dispersant was dispensed at the center of the oil slick in a flask. The flask was placed on an orbital shaker and shaken for 10 min at a rotation speed of $150 \mathrm{rpm}$. After shaking, the flask remained stationary for $10 \mathrm{~min}$. Then, $30 \mathrm{~mL}$ sample was collected and processed according to oil standard procedure. Control without adding dispersant and four replicate flasks were performed for quality control.

\subsubsection{Factors Affecting Dispersion}

One factor experiments were conducted to investigate the influence of every environmental factor on the dispersion effectiveness of the optimal dispersants. The factors and levels of each factor were as follows: temperature $(0,5$, 10,20 , and $\left.30^{\circ} \mathrm{C}\right)$, salinity $(0,5,10,20,30,35$, and 40 per thousand), $\mathrm{pH}(6,7,8,8.5,9$, and 10), DOR (1:10, $1: 15,1: 20,1: 25$, and $1: 30$ ). The rotational speed was kept constant at $150 \mathrm{rpm}$ for all dispersion experiments.

\subsubsection{Toxicity}

The acute toxicity of two optimal dispersants to fish was estimated by a short-term test on adult Danio rerio and Microgobius gulosus. Danio rerio obtained from local aquafarms and Microgobius gulosus caught from local shallow marine tide pools were raised in our lab in sterile fresh water and seawater at $25^{\circ} \mathrm{C}$, respectively. No food was provided before $24 \mathrm{~h}$ and during the test. On the day of experiment, test solution with concentration of dispersants $600 \mathrm{mg} / \mathrm{L}$ and control solution were prepared and aerated to restore the concentration of dissolved oxygen to air saturation value. 10 fish were then placed in the $5 \mathrm{~L}$ glass aquaria containing $3 \mathrm{~L}$ test and control solution. Experiments were carried out in duplicate. The number of dead was recorded after 1, 12, 24, and $48 \mathrm{~h}$.

\section{Results and Discussion}

\subsection{Formulation optimization}

The UD method is employed in this study because its principle is to replace the complete combination of experimental parameters by using relatively fewer experiment trials uniformly distributed within the parameter space, and emphasize the uniformity of space filling in experimental domain. In experiment trails, four kinds of surfactants were arranged according to uniform design table (Table 3). 24 baffled flask tests were performed, and two optimized dispersant formulations (No.13 and No.16) were identified. The DE of formulation No.13 and No.16 ranged from $30 \%$ to $60 \%$ from $5^{\circ} \mathrm{C}$ to $30^{\circ} \mathrm{C}$ at the DOR of 1:25 and $150 \mathrm{rpm}$ mixing speed. Under the same DOR and mixing speed, Corexit 9500, which was developed for dispersing heavy and weathered oils, its DE for heavy 
oil of IFO 180 and IFO 380 were both less than $30 \%$ at $5^{\circ} \mathrm{C}$ and $16^{\circ} \mathrm{C}$ [15]. Usually, hydrophilic- liphophilic-balance (HLB) is important in determining oil dispersion effectiveness and oil spill dispersants should traditionally have (HLB) values in the range of 10-15 [21]. The HLB values of formulation No.13 and No.16 were 13.37 and 12.49 , respectively, are in good agreement with the value of oil spill dispersant proposed. In addition, the mixture of nonionic and ionic surfactants solutions form mixed micelles exhibited better efficiency in decreasing oil-water interfacial tensions and lower critical micelle concentration (CMC) than individual components and facilitated the dispersion of the oil droplets; Meanwhile, the mixed surfactants and solvents systems also form a continuous film, which stabilizes the new interface and prevents the coalescence of oil droplets [22].

\subsection{Factors Affecting Dispersion}

The DE of oil spill dispersant depends on not only crude oil nature, but also various environmental conditions, such as mixing energy, temperature and salinity $[23,24]$. In order to optimize using conditions for the two dispersants developed in this study, the effects of DOR, temperature, salinity, and $\mathrm{pH}$ of seawater were investigated.

Figure 1(a) shows that the increase in DE as DOR in- creased from 1:30 to 1:10 for both formulations. The DE of formulation No.13 was sensitive to changes in DOR and decreased significantly when DOR was reduced to 1:25. However, DE of formulation No.16 decreased slowly with decrease of DOR. These results indicated that lower DOR (1:25 and 1:30) could be appropriate for dispersing this style of oil effectively and dramatically reduce the waste of dispersant. In addition, these two formulations had almost equal high DE when DOR was $1: 10$. It means that high DOR is in favor of decreasing oil-water interfacial tension and diluting the oil as small droplets into the water column. Formulation No.16 exhibits higher DE than that of formulation No.13 at the same DOR (from 1:15 to 1:30) suggested that formulation No.16 was superior to No.13 in this experiment condition.

Figure 1(b) shows temperature effects on the effectiveness of two dispersant formulations in dispersing QHD32-6 crude oil. It can be seen that DE of formulation No.13 and No.16 were high (> 40\%) at the low temperature $\left(5^{\circ} \mathrm{C}\right)$. This result indicated that these two dispersant formulations could be successfully applied as oil dispersant in cold weather. For formulation No.13, $\mathrm{DE}$ increased as temperature increased from 0 to $30^{\circ} \mathrm{C}$. Srinivasan, et al. [15] found that DE of three dispersants for IFO180 and IFO380 in the BFT were almost two times more effective at $16^{\circ} \mathrm{C}$ than at $5^{\circ} \mathrm{C}$ when mixing energy was sufficiently high. However, when the temperature increased from $5^{\circ} \mathrm{C}$ to $30^{\circ} \mathrm{C}$, DE declined for formulation No.16. Here, it is interesting to note that

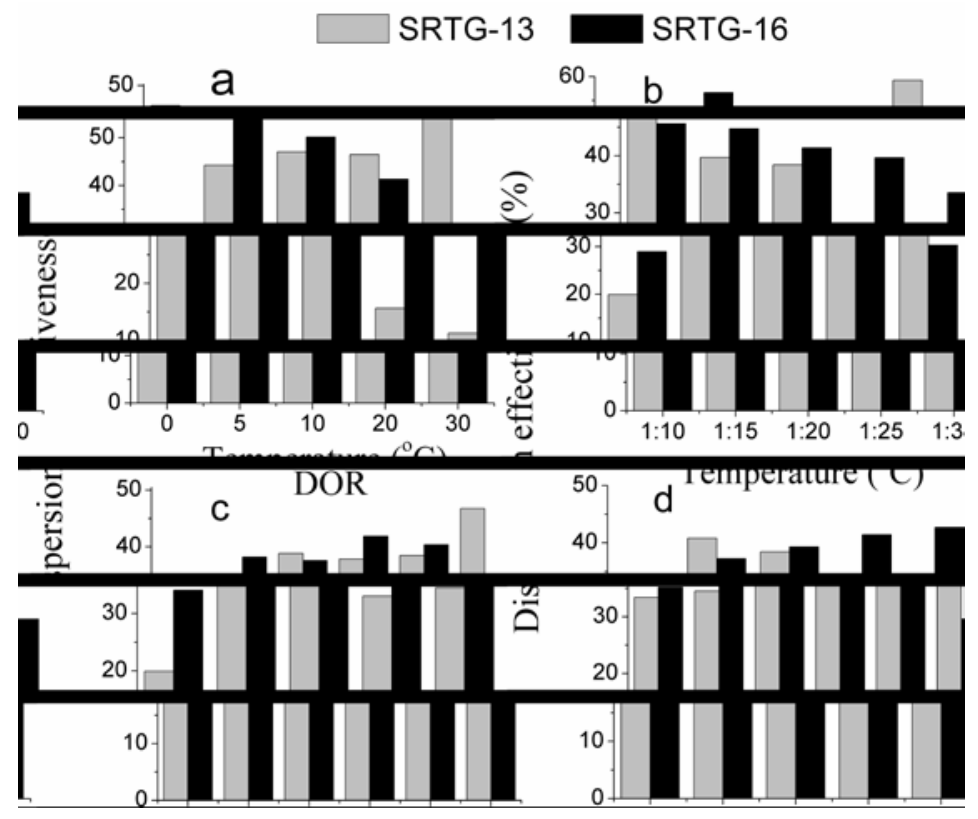

Figure 1. Effects of dispersant-to-oil ratio (DOR) (a), temperature (b, at 1:10 DOR), salinity (c, at 1:25 DOR), and pH (d, at 1:25 DOR) on dispersion effectiveness (DE) of formulation No.13 and No.16. The levels of fixed factors in every studied factor were consistent with seawater used in this study. 
high temperature exhibited negative effect on DE. The conflicting trends in DE with the increase in temperature have been observed by previous researchers $[25,26]$. It can be concluded that temperature may play a double role here: on the one hand, it decreases viscosity of the crude oil and thereby causes an increase in dispersion; on the other hand, it may change the physical properties of dispersants, which results the decrease of DE. A further study of the effect of temperature on DE should be performed to obtain clearer understanding the influencing mechanism.

Figure 1(c) shows that DE increased with a slowly increase in salinity from 10 to 40 per thousand for formulation No.13. As mentioned earlier, higher salinity increased the effectiveness of dispersants by preventing surfactant molecules from migrating into the water phase, equivalent to a salting-out effect for the surfactant from the saline solution [27]. This salting-out effect can promote association of surfactant molecules with oil at oilwater interfaces, which is important for lowering oilwater interfacial surface tensions in the oil-dispersant mixture [38]. For formulation No.16, results exhibited minimum DE when salinity was 40 per thousand, which may be caused by salting-in effect. Overall, salinity was not a significant factor affected DE of two formulations. Combined with the results of no detectable toxic effect of No.13 and No.16 on freshwater fish and saltwater fish, it can be concluded that these two dispersants are suitable for remediation of oil spill not only in fresh water but also in seawater.

The result in Figure 1(d) shows that DE of two formulations were both high when $\mathrm{pH}$ was in the range of $7-10$, which covered the values of most sea regions.

\subsection{Dispersant Toxicity}

Improvements in dispersant formulation mean that it is not only the increase of dispersion capability but also the decrease of toxicity. It was found that the landings of crustaceans had no any apparent reduction after spraying dispersant in Sea Empress incident off the coast of South Wales [29]. The field trials indicated that the use of dispersant was unlikely to have acute toxic effects on the marine environment. In this study, Danio rerio (freshwater fish) and Microgobius gulosus (saltwater fish) were used to study the acute toxicity of formulation No.13 and No.16. When these two types of fish were exposed in dispersant solution, lethality rates for Danio rerio at the end of 48 hours was 0 and for Microgobius gulosus, lethality rates was also 0 at the end of 24 hours. However, lethality rates for Danio rerio reached 60\% after exposed in one chemical dispersant (named GM) solution at the end of 12 hours. The toxicity results indicated that these two optimized dispersants formulations had lower toxicity and were in accordance with Chinese national standard [30], which requests lethality rates of these two types of fish to be no more than 50 percent at the end of 24 hours with the same exposure concentration of dispersant.

\section{Conclusion}

In this study, formula uniform design was successfully used to optimize dispersant formulations by arranging the levels of four surfactants continuously. Two optimized formulations were obtained in view of high dispersion effectiveness and low toxicity. The effects of environment factors on dispersant effectiveness were as below: First, the variation trend of DE increased with the increase of DOR was found for both of two optimized formulations. DE was still high when the formulation No.16 was used at low DOR. Using the dispersant at low DOR can save the usage amount of dispersant and reduce the possible damage of dispersant to environment. These two dispersant formulations could be also effective in response of oil spill in cold weather. Secondly, saltingout effect of salinity lowered oil-water interfacial surface tensions and promoted the dispersion of oil, but the effect was not significant. Similarly, $\mathrm{pH}$ was not a significant factor affected DE of two formulations, which were both high when $\mathrm{pH}$ was in the range of 7-10. Finally, these two optimized dispersants formulations had lower toxicity towards two kinds of fish (Danio rerio and Microgobius gulosus). Nonetheless, whether the two optimized dispersant formulations could be introduced to apply in oil spill response requires a further field test to better understand the effects of oil properties, interaction of environmental factors and detailed toxicity.

\section{Acknowledge}

The authors wish to acknowledge the insightful comments and suggestions from the anonymous reviewers and editors for improving the content of this manuscript. The research was funded by China Offshore Environmental Service Ltd. (2010098).

\section{REFERENCES}

[1] J. Haapkylal, F. Ramade and B. Salvat, "Oil Pollution on Coral Reefs: A Review of the State of Knowledge and Management Needs," Life and Environment, Vol. 57, No. 1-2, 2007, pp. 91-107.

[2] E. M. Chuvilin and E. S. Miklyaeva, “An Experimental Investigation of the Influence of Salinity and Cryogenic Structure on the Dispersion of Oil and Oil Products in Frozen Soils," Cold Regions Science and Technology, Vol. 37, No. 2, 2003, pp. 89-95. doi:10.1016/S0165-232X(03)00063-6 
[3] J. S. Poland, M. J. Riddle and B. A. Zeeb, “Contaminants in the Arctic and the Antarctic: A Comparison of Sources, Impacts, and Remediation Options," Polar Record, Vol. 39, No. 4, 2003, pp. 369-383. doi:10.1017/S0032247403002985

[4] D. P. Hoult, "Oil Spreading on the Sea," Annual Review of Fluid Mechanics, Vol. 4, No. 1, 1972, pp. 341-368. doi:10.1146/annurev.fl.04.010172.002013

[5] P. S. Daling, I. Singsaas, M. Reed and O. Hansen, "Experiences in Dispersant Treatment of Experimental Oil Spills,” Spill Science \& Technology Bulletin, Vol. 7, No. 5-6, 2002, pp. 201-213. doi:10.1016/S1353-2561(02)00061-0

[6] A. Lewis, B. Ken Trudel, R. C. Belore and J. V. Mullin, "Large-scale Dispersant Leaching and Effectiveness Experiments with Oils on Calm Water," Marine Pollution Bulletin, Vol. 60, No. 2, 2010, pp. 244-254. doi:10.1016/j.marpolbul.2009.09.019

[7] P. J. Brandvik and P. S. Daling, "Optimisation of Oil Spill Dispersant Composition by Mixture Design and Response Surface Methods," Chemometrics and Intelligent Laboratory Systems, Vol. 42, No. 1-2, 1998, pp. 63-72. doi:10.1016/S0169-7439(98)00009-4

[8] A. M. Al-Sabagh, S. H. El-Hamouly, A. M. Atta, M. R. N. El-Din and M. M. Gabr, "Synthesis of Some Oil Spill Dispersants Based on Sorbitol Esters and Their Capability to Disperse Crude Oil on Seawater to Alleviate Its Accumulation and Environmental Impact," Journal of Dispersion Science and Technology, Vol. 28, No. 5, 2007, pp. 661-670. doi:10.1080/01932690701341751

[9] N. P. Ventikos, E. Vergetis, H. N. Psaraftis and G. Triantafyllou, "A High-level Synthesis of Oil Spill Response Equipment and Countermeasures,” Journal of Hazardous Materials, Vol. 107, No. 1-2, 2004, pp. 51-58. doi:10.1016/j.jhazmat.2003.11.009

[10] R. R. Lessard and G. Demarco, "The Significance of Oil Spill Dispersants,” Spill Science \& Technology Bulletin, Vol. 6, No. 1, 2000, pp. 59-68. doi:10.1016/S1353-2561(99)00061-4

[11] R. Stakenienè and K. Jokšas, "Dispersants and Their Influence on Oil Spread in Water Bodies," Environmental research, engineering and management, Vol. 4, No. 34, 2004, pp. 61-67.

[12] M. M. Singer, S. George, S. Jacobson, I. Lee, L. L. Weetman, R. S. Tjeerdema and M. L. Sowby, "Acute Toxicity of the Oil Dispersant Corexit 9554 to Marine Organisms," Ecotoxicology and Environmental Safety, Vol. 32, No. 1, 1995, pp. 81-86. doi:10.1006/eesa.1995.1088

[13] Cutter Information Corp., "The Dispersant Use Decision-making Process,” In: Oil Spill Intelligence Report, White Paper Series, April, 1998.

[14] Fiocco, R.J, P.S. Daling, G. DeMarco, R.R. Lessard and G.P.Canevari. 1999b. Chemical Dispersibility Study of Heavy Bunker Fuel Oil. Proc. 22nd Arctic and Marine Oilspill Program, Environment Canada, Ottawa, Canada, pp 173-186.
[15] R. Srinivasan, Q. Lu, G. A. Sorial, A. D. Venosa and J. Mullin, "Dispersant Effectiveness of Heavy Fuel Oils Using Baffled Flask Test,” Environmental Engineering Science, Vol. 24, No. 9, 2007, pp. 1307-1320. doi:10.1089/ees.2006.0251

[16] A. D. Venosa, D. W. King and G. A. Sorial, “The Baffled Flask Test for Dispersant Effectiveness: A Round Robin Evaluation of Reproducibility and Repeatability," Spill Science \& Technology Bulletin, Vol. 7, No. 5-6, 2002, pp. 299-308. doi:10.1016/S1353-2561(02)00072-5

[17] X. Wang, L. Gong, S. Liang, X. Han, C. Zhu and Y. Li, "Algicidal Activity of Rhamnolipid Biosurfactants Produced by Pseudomonas aeruginosa," Harmful Algae, Vol. 4, No. 2, 2005, pp. 433-443. doi:10.1016/j.hal.2004.06.001

[18] D. Song, S. Liang and J. Wang, "Structure Characterization and Physic-chemical Properties of Sophorolipid Biosurfactants," Environmental chemistry (in Chinese with English abstract), Vol. 30, No. 8, 2011, pp. 1474- 1479.

[19] K. T. Fang, "The Uniform Design: Application of Number-theoretic Methods in Experimental Design,” Acta Mathematicae Applicatae Sinica, Vol. 3, 1980, pp. 363-372.

[20] S. Chandrasekar, G. A. Sorial and J. W. Weaver, "Dispersant Effectiveness on Three Oils under Various Simulated Environmental Conditions," Environmental Engineering Science, Vol. 22, No. 3, 2005, pp. 324-336. doi:10.1089/ees.2005.22.324

[21] P. Riley, "Compositions and Methods for Dispersing and Biodegrading Spilled Petroleum Oils and Fuels,” PetroTech, Patent number: 5753127, 1998.

[22] S. A. Mahmouda and T. M. Abd El-Rahmanb, "A New Family of Oil Spill Dispersants: Part 1. The Effect of Structural Variation on the Oil Spill Dispersant Efficiency,” Petroleum Science and Technology, Vol. 28, No. 14, 2010, pp. 1394-1406. doi:10.1080/10916460802637585

[23] B. Mukherjee and B. A. Wrenn, "Influence of Dynamic Mixing Energy on Dispersant Performance: Role of Mixing Systems,” Environmental Engineering Science, Vol. 26, No. 12, 2009, pp. 1725-1737. doi:10.1089/ees.2009.0159

[24] B. Mukherjee and B. A. Wrenn, "Effects of Physical Properties and Dispersion Conditions on the Chemical Dispersion of Crude Oil," Environmental Engineering Science, Vol. 28, No. 4, 2011, pp. 263-273. doi:10.1089/ees.2010.0131

[25] D. C. Byford, P. J. Green and A. Lewis, "Factors Influencing the Performance and Selection of Low-temperature Dispersants," Proceedings of the Sixth Arctic Marine Oil Spill Program, Edmonton, Canada, 1983.

[26] M. F. Fingas, "Dispersants: A Review of Effectiveness Measures and Laboratory Physical Studies,” Ottawa, Ontario: Environmental Emergencies Technology Division, Environment Canada, 1991.

[27] D Mackay, A. Chau, K. Hossain and M. Bobra, "Measurement and Prediction of the Effectiveness of Oil Spill 
Chemical Dispersants,” In Oil Spill Chemical Dispersants, Research, Experience and Recommendations, STP 840. Tom E. Allen, Ed., American Society for Testing and Materials, Philadelphia, 1984, pp. 38-54.

[28] S. Chandrasekar, G. A. Sorial and J. W. Weaver, "Dispersant Effectiveness on Oil Spills - impact of Salinity," ICES Journal of Marine Science, Vol. 63, No. 8, 2006, pp. 1418-1430.

\section{doi:10.1016/j.icesjms.2006.04.019}

[29] H. Chapman, K. Purnell, R. J. Law and M. F. Kirby, “The Use of Chemical Dispersants to Combat Oil Spills at Sea: A review of practice and research needs in Europe,” $\mathrm{Ma}$ rine Pollution Bulletin, Vol. 54, No. 7, 2007, pp. 827-838. doi:10.1016/j.marpolbul.2007.03.012

[30] GB 18188.1-2000, Oil Spill Dispersant-Technical Regulations (in Chinese). 